\title{
GIS-based modeling of rainfall-induced landslides using data mining-based functional trees classifier with AdaBoost, Bagging, and MultiBoost ensemble frameworks
}

\begin{abstract}
The main objective of this study is to propose and verify a novel ensemble methodology that could improve prediction performances of landslide susceptibility models. The proposed methodology is based on the functional tree classifier and three current state-of-the art machine learning ensemble frameworks, Bagging, AdaBoost, and MultiBoost. According to current literature, these methods have been rarely used for the modeling of rainfall-induced landslides. The corridor of the National Road 32 (Vietnam) was selected as a case study. In the first stage, the landslide inventory map with 262 landslide polygons that occurred during the last 20 years was constructed and then was randomly partitioned into a ratio of 70/30 for training and validating the models. Second, ten landslide conditioning factors were prepared such as slope, aspect, relief amplitude, topographic wetness index, topographic shape, distance to roads, distance to rivers, distance to faults, lithology, and rainfall. The model performance was assessed and compared using the receiver operating characteristic and statistical evaluation measures. Overall, the FT with Bagging model has the highest prediction capability $(\mathrm{AUC}=0.917)$, followed by the FT with MultiBoost model $(\mathrm{AUC}=0.910)$, the FT model $(\mathrm{AUC}=0.898)$, and the FT with AdaBoost model $(\mathrm{AUC}=0.882$ ). Compared with those derived from popular methods such as J48 decision trees and artificial neural networks, the performance of the FT with Bagging model is better. Therefore, it can be concluded that the FT with Bagging is promising and could be used as an alternative in landslide susceptibility assessment. The result in this study is useful for land use planning and decision making in landslide prone areas.
\end{abstract}

Keyword: Landslide; GIS; Functional trees; AdaBoost; MultiBoost; Bagging; Vietnam 